\title{
Colloid Approach to the Sustainable Top-Down Synthesis of Layered Materials
}

\author{
Housseinou Ba, Lai Truong-Phuoc, Cuong Pham-Huu, Wen Luo, Walid Baaziz, Thierry Romero, \\ and Izabela Janowska*๑
}

Institut de Chimie et Procédés pour l'Énergie, l'Environnement et la Santé (ICPEES), CNRS UMR 7515-University of Strasbourg, 25 rue Becquerel, 67087 Strasbourg, France

Supporting Information

ABSTRACT: The successful future of $2 \mathrm{D}$ materials, which are crucial for accelerating technology development and societal requirements, depends on their efficient preparation in an economical and ecological way. Herein, we present a significant advance in the top-down exfoliation and dispersion method via an aqua colloid approach. We demonstrate that a broad family of natural oil-in-water emulsification agents with an elevated hydrophilic/lipophilic balance acts in the exfoliation of layered materials and the formation of their concentrated colloids. The concentration exceeds $45 \mathrm{~g} / \mathrm{L}$ for exfoliated few-layered graphene sheets possessing a micrometer size. The exfoliation of carbon nanofibers provides one of the best known unsupported and $\mathrm{N}$ undoped metal-free catalysts to date in the selective dehydrogenation of ethylbenzene to styrene. Other examples include aqua colloids of exfoliated/dispersed nitrides, carbides, or nanodiamonds.

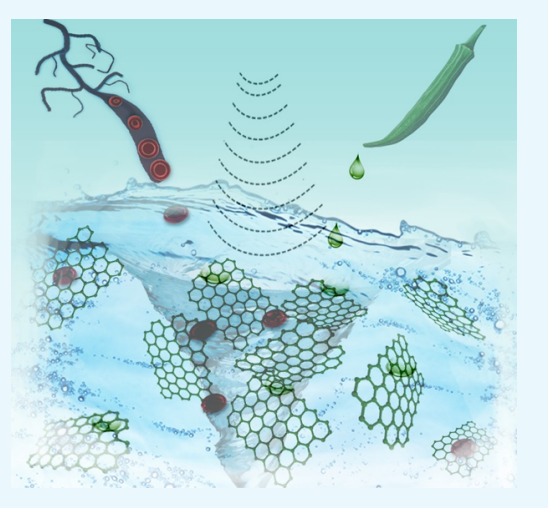

\section{INTRODUCTION}

The accelerating technological development and societal energy demands require rapid and firm solutions that have to match the economical and environmental issues. The major challenges concern several aspects, starting with the choice of crucial materials and their preparation methods. Because of their planar geometry and related in-plane propagation properties, 2D materials and especially (few-layered) graphene (FLG) have become materials of choice. In applications for which a high yield of thin-layered structures is required, the top-down methods based on exfoliations of bulk materials are of interest, especially when more than one layer in the final material is sufficient and/or preferable. ${ }^{1}$ This is especially true for liquid-exfoliated FLG to be next applied in composites or energy storage among others. Additionally, the related solution-processable suspensions with significant $2 \mathrm{D}$ material concentration are a central interest point in coating or printing sectors. ${ }^{1,2}$ A highest concentration of graphene layers, up to $38 \mathrm{~g} / \mathrm{L}$, has been obtained in $\mathrm{N}$-methyl-2pyrrolidone, a performance assigned to the appropriate surface tension $(\sim 40 \mathrm{mN} / \mathrm{m}))^{2,3}$ The advantages of processing in aqueous media are, however, environmentally unquestionable. For this purpose and because of the hydrophobic character of graphite (or other materials), graphene production runs either via graphene oxide synthesis, followed by hardly happening conjugated $\mathrm{C}=\mathrm{C}$ lattice restoration, ${ }^{4,5}$ or via the use of surfactants. The recent trends in surfactants register some biologically derived species such as enzymes, ${ }^{6}$ RNA or DNA, ${ }^{7,8}$ vitamin B derivatives, ${ }^{9}$ proteins, ${ }^{10-12}$ polysaccharides, ${ }^{13,14}$ carbohydrates, ${ }^{15}$ or polyphenols. ${ }^{16}$ Singular examples reach high-concentration aqua suspension of graphene using ionic surfactants. ${ }^{9,17}$ These biosurfactants, especially proteins such as bovine serum albumin (BSA), were revealed to be efficient exfoliation agents, and investigations of BSA adsorption over graphitic surface have been undertaken. ${ }^{11,17}$ Aiming to diminish defects/oxygen content and enhance the process yield, the viaprotein exfoliation was accompanied by shear/turbulence in a kitchen blender. ${ }^{12,18}$ Although a concentration of BSA-exfoliated FLG in water up to $7 \mathrm{~g} / \mathrm{L}$ was achieved, FLG sheets with a weakly defined geometry, often crumpled, and with a low average size $(\sim 0.3 \mu \mathrm{m})$ were observed. ${ }^{12}$ Most of the other biosurfactantassisted exfoliations of graphite provide a similar relatively low sheet size. Such a low sheet size with enhanced oxygen content (edges) is beneficial for some applications including biomedical $^{9,20}$ or catalysis, ${ }^{21,22}$ but not for domains where large size sheets are better suited for easy propagation of a given property within the bulk or on the surface of the final materials as nanocomposites. The structure-property-application relationship is indeed a crucial point, and a given property depends on the way graphene is arranged and tailored in terms of geometry and chemistry. ${ }^{23}$

Our present work aims not only to push the limits of exfoliation of laminar materials in water but also to open new potential horizons for future development. We do not take chemistry to a kitchen but gastronomy to a laboratory. Although the effects of hydrophobic and hydrophilic regions in biomolecules such as BSA on the production of stable aqua dispersion was investigated previously, ${ }^{11,17,24}$ we take a broader look on it. On the basis of our results, we define the exfoliation

Received: September 5, 2017

Accepted: November 21, 2017

Published: December 4, 2017 
ability of proteins such as BSA or hemoglobin (HEM) simply by their high hydrophilic/lipophilic balance (HLB) and related oilin-water ("o/w") emulsification ability as a driving force of the exfoliation, dispersion, and stabilization of hydrophobic 2D materials in water. ${ }^{25}$ The approach meets the principles on which the use of BSA and HEM in gastronomy, for instance, is based. ${ }^{26}$ According to this concept, several other "comestible", biocompatible, and naturally existing emulsifiers with sufficiently high molecular systems such as maltodextrin, gums, agar-agar, or gombo extracts have successfully been tested by us. Of course, the $\mathrm{o} / \mathrm{w}$ emulsification process includes the adsorption of specific groups from HLB systems on the material surface, but the adsorbing groups will differ in HLB systems; for example, the benzene rings or disulfide groups from BSA as described earlier are absent in maltodextrin or other polysaccharides.

From a kitchen, we take also "the emulsion homogenization concept" and add turbulence forces (mixing) to ultrasonications. This simple innovation allows the efficient exfoliation of expanded graphite (EG) and provides the aqua colloids/ emulsions, with concentrations in FLG higher than $45 \mathrm{~g} / \mathrm{L}$, where sheets of FLG flakes are of several micron size. ${ }^{27}$ The emulsification property of HLB systems demonstrates that, apart from the typical laminar graphite, boron nitride or carbon nitride materials, silicon carbide, and nanodiamonds can form stable aqua colloids. However, we essentially focus on EG and carbon nanofibers (CNFs), first, to reach conductive large micrometersized sheets and, second, to test exfoliation on CNFs for catalysis purpose. The latter CNF-exfoliation-originated carbon reveals metal-free catalytic activity in the selective dehydrogenation of ethylbenzene to styrene with a performance exceeding that of nanodiamonds, the highest to date (without considering $\mathrm{N}$ doped and supported catalysts). ${ }^{22}$

\section{RESULTS AND DISCUSSION}

In general, $x \mathrm{mg}$ of initial materials such as EG and $y=0.1 x \mathrm{mg}$ of HLB active natural system are added to water and all is subjected to probe ultrasonication treatment assisted by mixing with a "standard" stirring magnetic bar (a mechanically driven top stirring can also be applied with a bath sonication). The optimal ratio of the initial material and HLB was chosen considering a yield of exfoliation for colloids with a low concentration $(1 \mathrm{~g} / \mathrm{L})$. Figure $1 \mathrm{a}, \mathrm{b}$ demonstrates the representative aqua colloids of FLG-BSA obtained after the ultrasonication/mixing of EG (40 $\mathrm{g} / \mathrm{L})$ and of graphite $(30 \mathrm{~g} / \mathrm{L})$, respectively. In the case of EG, an additional amount of EG (and BSA) is subsequently added, giving a concentration of $54 \mathrm{~g} / \mathrm{L}$. The settling-down process occurring over $24 \mathrm{~h}$ results finally in stable colloids with concentrations of $47 \mathrm{~g} / \mathrm{L}$ for EG and $8 \mathrm{~g} / \mathrm{L}$ for graphite. Such high concentrations of exfoliated materials, especially for EG, are possible because of the coupling of HLB system and ultrasonication/mixing. The addition of mixing to ultrasonication helps to homogenize the process in general and especially to unify the contact between overall reaction medium and ultrasound. This is not a case of bath or tip ultrasonications, in which when used alone, the applied sound intensities are not uniform and already dissipate at a distance of $1 \mathrm{~cm}^{-1}$ from the tip in the tip sonicators. ${ }^{28-30}$ On the other hand, mixing alone is insufficient for efficient exfoliation, but when coupled with sonication, it also impacts the adsorption of HLB on the graphite surface and solvation of the hydrophylic groups of HLB systems by water to overcome the van der Waals interlayer forces in graphite and thus efficiently disperse the graphite sheets. Moreover, in addition to its hydrophobic character, EG is very
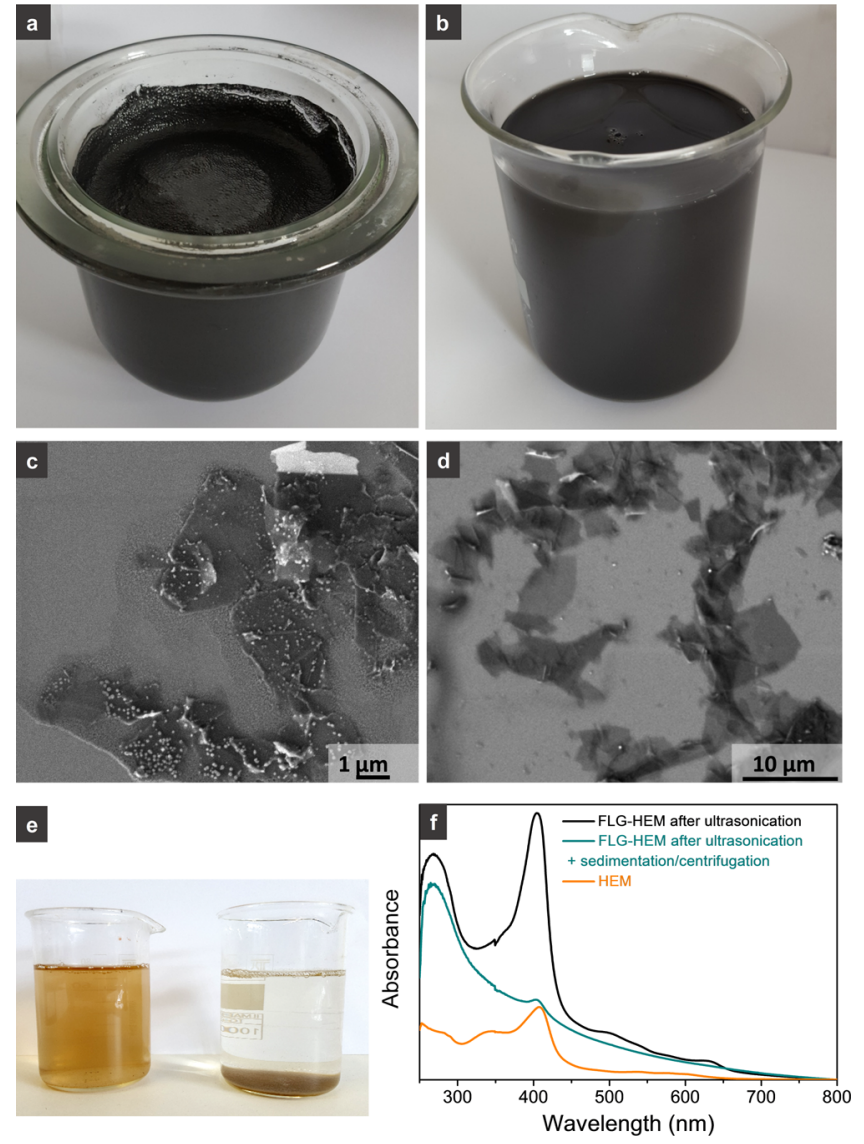

Figure 1. (a) Colloid of FLG-BSA in water obtained after the ultrasonication of EG with a concentration of around $40 \mathrm{~g} / \mathrm{L}$, (b) colloid of FLG-BSA in water obtained after the ultrasonication of graphite (pellets) with a concentration of around $30 \mathrm{~g} / \mathrm{L},(\mathrm{c}, \mathrm{d})$ scanning electron microscopy (SEM) micrographs of the products obtained after the ultrasonication and separation of FLG-HEM in water: (c) sediment fraction and (d) supernatant fraction, (e) optical photo of the HEMwater colloid before ultrasonication and after ultrasonication and sedimentation, and (f) UV-vis spectra of aqua colloids of HEM and FLG-HEM before and after separation process (sedimentation or centrifugation).

lightweight and simply remains on the water surface during the sonication. The addition of stirring creates a vortex, which rapidly and progressively aspires EG (Supporting Information, Figure S1). Apart from highly concentrated colloids and exfoliation efficiency, the use of EG is essential to achieve a large size of FLG flakes. Instead of few hundred nanometers $(300 \mathrm{~nm})$ reported previously from graphite, mainly few-micrometer large sheets can be observed after a relatively short time $(2 \mathrm{~h})$ of ultrasonication/mixing. The effect of the size of flakes is very important for several applications including composites and coating layer fields. $^{31,32}$ (A very recent work reported the use of microfluidization method for the efficient exfoliation of graphite in water, where relatively thick and medium-size FLG flakes were obtained. $)^{30}$ Herein, a high, around few thousands, aspect ratio of FLG ( 2000 considering the $3 \mu \mathrm{m}$ flake of five graphene sheets) is calculated.

The FLG-BSA colloid with $47 \mathrm{~g} / \mathrm{L}$ can be also diluted, if necessary. Progressive dilution induces a slight aggregation of FLG-BSA at dilution by $10^{3}(0.047 \mathrm{~g} / \mathrm{L})$, which can be rapidly overcome by the addition of BSA grain and mild redispersion (Figure S2, Supporting Information). This shows that there is 

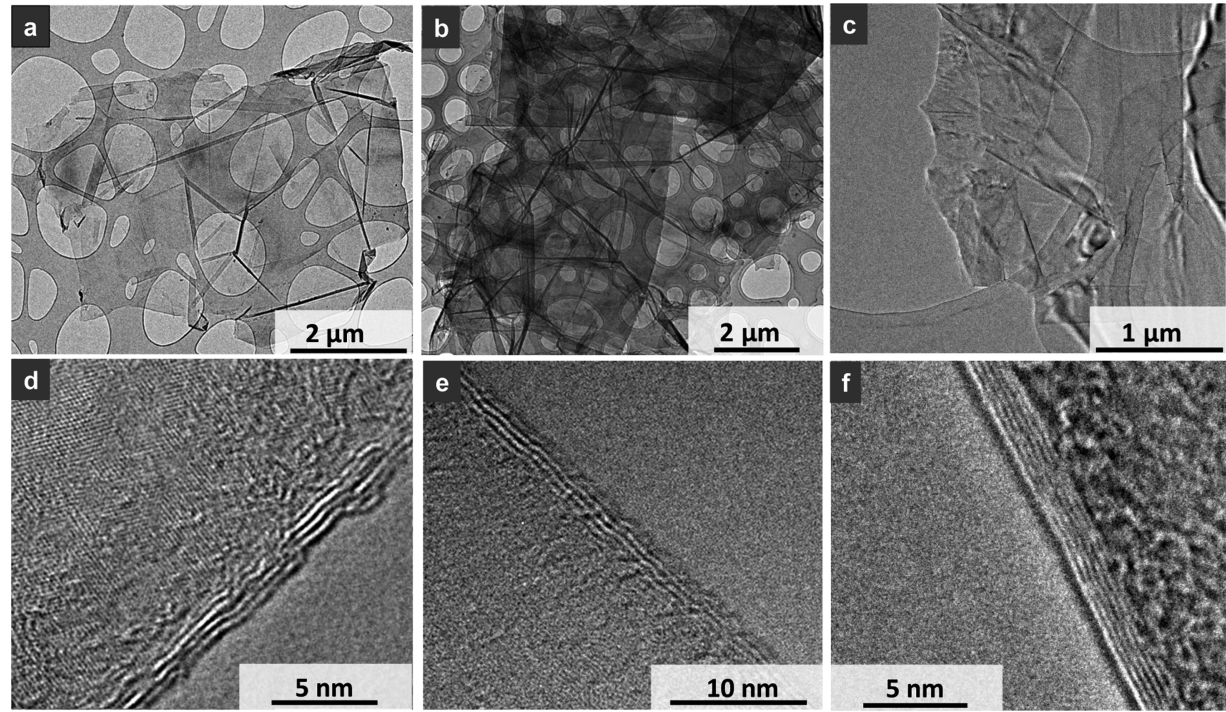

Figure 2. $(a-c)$ Representative TEM micrographs of FLG obtained after FLG-HLB-water system ultrasonication, where HLB is BSA, maltodextrin, myoglobin, respectively, and $(\mathrm{d}-\mathrm{f})$ number of sheets, four, four, and six, observed at the curved edges in FLG-BSA, FLG-HEM, and FLG-agar-agar, respectively. (For comparison with the literature data, most of the presented investigations concern the two globin-assisted exfoliations. Yet, detailed studies of other mentioned biosystems are necessary in the near future.)
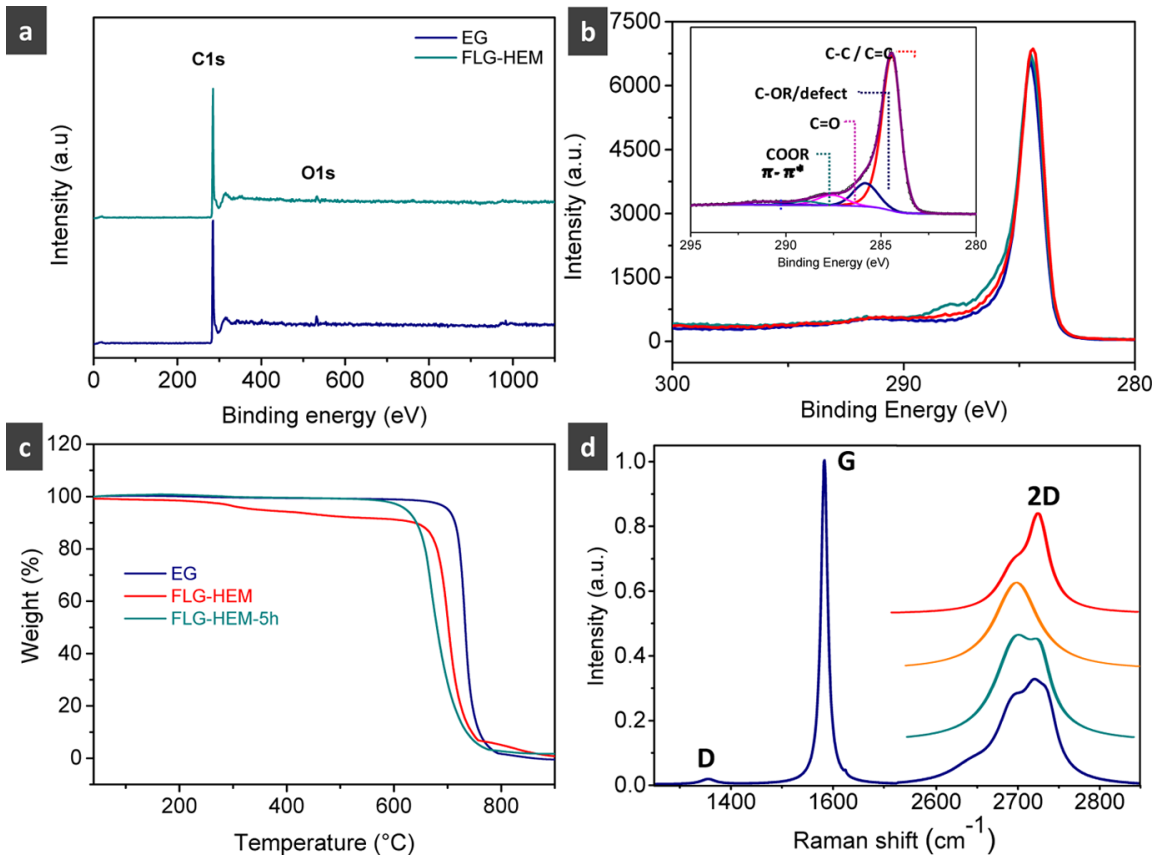

Figure 3. (a) General XPS spectra of EG and FLG-HEM, (b) C 1s XPS spectra of EG (blue), FLG-HEM (red), and FLG-HEM-5h (green) (inset: deconvoluted C 1s spectrum of FLG-HEM), (c) TGA curves of EG, FLG-HEM, and FLG-HEM-5h, and (d) full Raman spectrum of FLG flake and $2 \mathrm{D}$ peaks recorded on several FLG flakes (a laser excitation wavelength of $532 \mathrm{~nm}$ ).

still room to improve the stabilization of colloids by HLB systems and possibly their mixtures and to establish the optimal parameters related to the emulsification equilibrium. The emulsification phenomena can already be observed for ultrasonicated aqua suspensions of pure HEM and BSA proteins, where, according to the biochemistry literature, the formation of microspheres (microcapsules) occurs. ${ }^{33,34}$ Such a formation has origins in the sonolysis of water, during which the acoustic cavitations produce hydrogen and hydroxyl radicals and, in the presence of oxygen, a superoxide radical. The latter plays subsequently the role of cross-linking agent between disulfide bonds from cysteine residues in microcapsules. ${ }^{33-35}$ Such spheres of around 60-150 nm are also observed through microscopic analysis in graphite-protein sonolysis product, and their inferior dimension could be affected by partial protein degradation, by mixing, or finally by interactions with graphite. The spheres are separated together with heavy and weakly exfoliated fraction of graphite through a $24 \mathrm{~h}$ precipitation/ decantation step (Figure 1c) and are quasi-absent in the stable supernatant fraction (Figure 1d). In the latter, the globin residue is present in amount sufficient to glue and link the FLG flakes and to form stable colloids. The separation of unstable HEM species from stable colloidal HEM can be clearly observed for pure HEM ultrasonically dispersed in water. In this case, a high amount of 

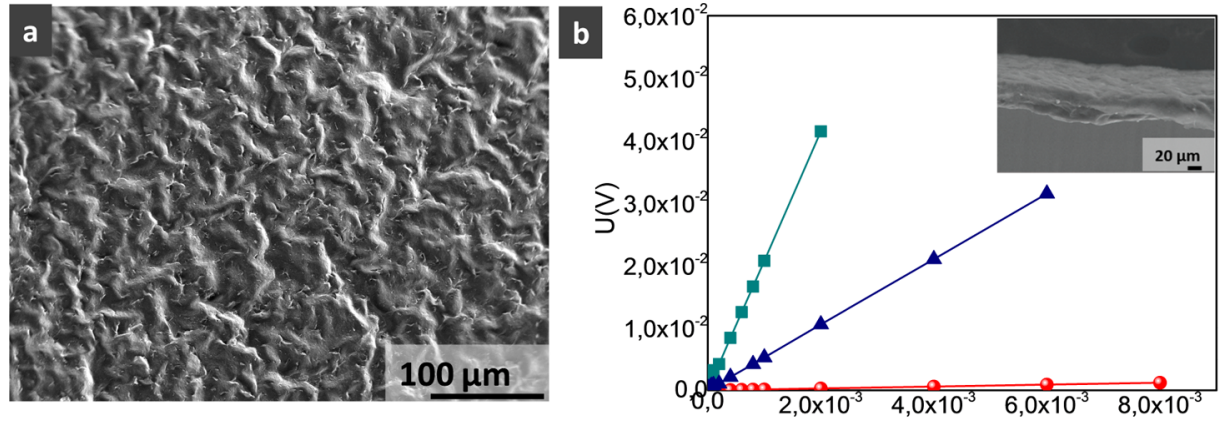

$\mathrm{I}(\mathrm{A})$
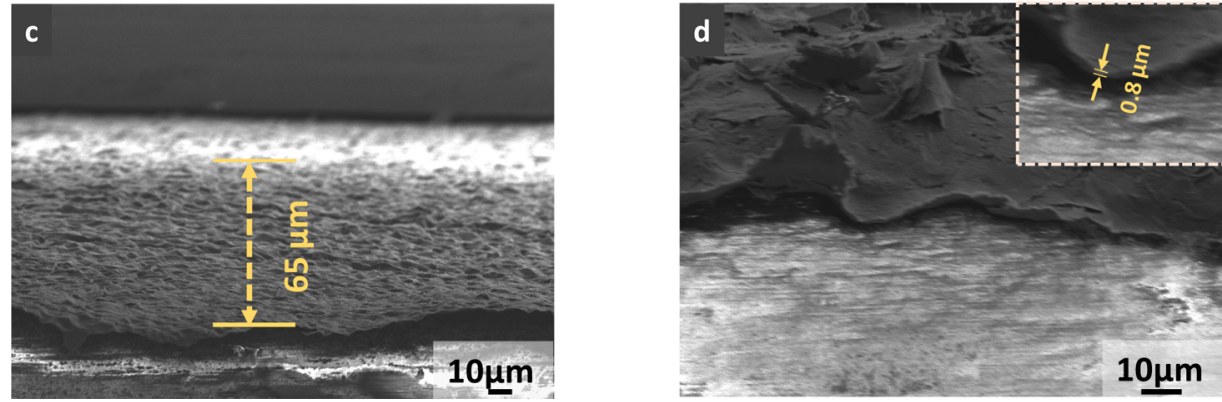

Figure 4. (a) SEM micrograph of the surface of the FLG-BSA paper; (b) representative $I-V$ curves measured for FLG-protein papers before (green) and after annealing and FLG after acid treatment and annealing (blue and red); and related SEM micrographs showing the thickness and transversal morphology of the papers: (b inset and c) FLG-BSA, (d) FLG-acid.

HEM settles down within a few hours (Figure 1e) and it seems that the presence of FLG and subsequent interactions between FLG-HEM-water, vice versa, increase the stability of HEM in water. The residue of HEM in the suspension can be easily followed by UV-vis spectroscopy as presented here for the FLG-HEM suspension (Figure 1f). In the case of BSA, the precipitation and formation of spheres after ultrasonication/ mixing are significantly weaker as the protein is quasi-dissolved in water (Figure S3, Supporting Information). The stability behavior of BSA in water, as mentioned above, remains unchanged in the case of the FLG-BSA colloid, and BSA demonstrates a superior ability for EG dispersion compared to HEM. This is probably related to the better HLB of BSA and related detergent-like property. ${ }^{26,36}$ The slight foam formation on the colloid/air surface can be observed in Figure 1a. It may be reminded that with an increase of HLB (from $\sim 8$ ), the $\mathrm{o} / \mathrm{w}$ emulsification ability changes from external $\mathrm{o} / \mathrm{w}$ phase formation to complete solubility of o/w through detergent solutions for $\mathrm{HLB} \approx 13-15$. Because of the presence of relatively heavy iron and large porphyrin systems, the impact of the hydrophilic part is reduced in HEM. The translucent nature of the HEM-water system and lucent (soluble) BSA-water confirm the different HLB for both proteins (Figures 1e and S3, Supporting Information).

Figure 2 shows the representative transmission electron microscopy (TEM) micrographs of FLG sheets obtained after the exfoliation of EG assisted by BSA, HEM, maltodextrin, myoglobin, and agar-agar, after ultrasonication/mixing for $2 \mathrm{~h}$. According to TEM and SEM analyses, the average size of the FLG flakes reaches several micrometers (Figures $1 \mathrm{~d}$ and $2 \mathrm{a}-\mathrm{c}$ ). The TEM micrographs obtained from the analysis at the edges of flakes reveal the presence of few sheets, five in average (Figure 2d-f).

Raman and X-ray photoelectron spectroscopies (XPS), thermal gravimetric analysis (TGA), and conductivity measurements of FLG-HEM confirm the quite high quality of the sample. Micro-Raman spectra recorded for several FLG-HEM flakes show typical D, G, and $2 \mathrm{D}$ vibration peaks at 1356,1583 , and $2720 \mathrm{~cm}^{-1}$, respectively (Figure $3 \mathrm{~d}$ ). The relatively weak D peak indicates the low defect content, and the $I_{\mathrm{D}} / I_{\mathrm{G}}$ ratio calculated for several flakes varies within the range of $0.02-0.08$. This variation arises from the variable number of sheets and amount of HEM on the FLG surface. The shift of the 2D peaks toward lower wavenumbers associated with the broadening of the higher energy 2D subpeaks, observed for several flakes, confirms some distribution of the FLG-HEM thickness with an excess of low sheet numbers, less than $5 .^{37}$

Apart from FLG sheets of significant size and low defect content, the exfoliation/dispersion process can be prolonged to decrease the size of the sheets and enhance their decoration with oxygen (Figure S4, Supporting Information). For this purpose, the ultrasonication/mixing was extended to $5 \mathrm{~h}$ (FLG-HEM5h): a significant degradation/cutting of FLG induced by microjets and shock waves was expected. Two mechanisms can be proposed for the cavitation near the carbon surface, which creates localized erosion and/or oxygen- and hydrogen-based radical formation during the sonolysis of water. ${ }^{35}$

The general XPS spectra confirm the high purity of the samples (Figure 3a), and the defects as well as oxygen content increase with sonolysis time, as observed by XPS (Figure $3 \mathrm{~b}$ and Table S1, Supporting Information) and TGA data (Figures 3c and S5, Supporting Information). The full width at halfmaximum of the $\mathrm{C} 1 \mathrm{~s}$ peak and oxygen-to-carbon ratio for $\mathrm{EG}$, FLG-HEM, and FLG-HEM-5h increase progressively and are $1.18,1.21$, and 1.25 and $0.024,0.039$, and 0.090 , respectively. Accordingly, the $\pi-\pi^{*}$ transition peaks decrease, indicating a lower electronic delocalization. A decrease of combustion temperature in TGA is clear for FLG-HEM and FLG-HEM$5 \mathrm{~h}$ compared to EG. (FLG-HEM starts to burn at a low temperature because of the locally enhanced amount of HEM, whereas the main combustion temperature of FLG-HEM is higher than that of FLG-HEM-5h, according to lower oxygen/ 


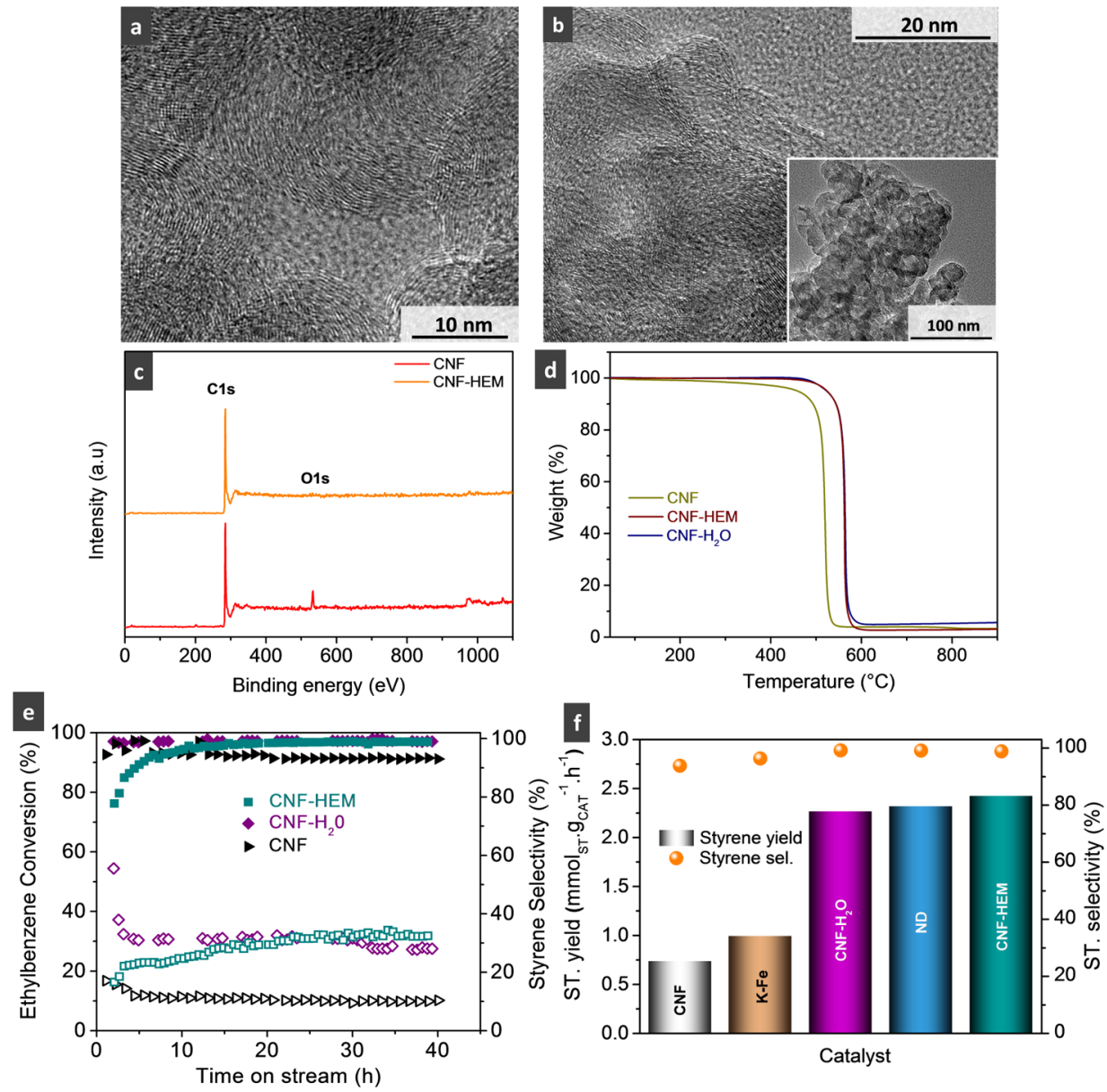

Figure 5. (a,b) TEM micrographs of the CNF-HEM catalyst obtained through the exfoliation of CNFs in the HEM-water system, (c) general XPS spectra of CNF and CNF-HEM, (d) TGA curves of CNF before and after exfoliation/cutting in water, (e) ethylbenzene conversion (open symbols) and styrene selectivity (filled symbols) as a function of time on stream obtained for CNF-HEM (green), CNF- $\mathrm{H}_{2} \mathrm{O}$ (violet), and CNF (black) catalysts, and (f) benchmarking of the activities of the catalysts prepared herein with those of commercial and nanodiamond catalysts in the selective dehydrogenation of ethylbenzene to styrene.

defect content.) To check the impact of the protein on electrical conductivity, FLG-HEM and FLG-BSA as well as pure FLG after the acid hydrolysis of the proteins (FLG-acid) were measured and calculated using the four-point probe (FPP) method measurements. The measurements were performed on papers formed by the filtration of the FLG-protein supernatants and FLG-acid suspension in iso-propanol (Figure S6, Supporting Information). The papers were also subjected to an annealing process at $700{ }^{\circ} \mathrm{C}$ in $\mathrm{He}$ (Figure 4a,b). According to the average thickness of the papers determined by SEM, the calculated conductivities of the FLG-protein papers are roughly $10^{2} \mathrm{~S} / \mathrm{m}$ order before annealing and $10^{4} \mathrm{~S} / \mathrm{m}$ order after annealing. A conductivity of $10^{5} \mathrm{~S} / \mathrm{m}$ order is reached for the FLG treated with acid and annealed; thus, it is free of HLB (FLG-acid). The superior conductivity of FLG-acid papers can be related, apart from protein removal, to the beneficial transversal paper morphology, where FLG flakes are closely packed, ensuring their efficient interconnection (Figure 4d). This is not a case for FLG-protein papers and especially for BSAassisted exfoliation samples, where a spongelike arrangement of the flakes can be observed, because of the detergent nature of the globin (Figure 4c).

Apart from "3D" graphite, we applied the present exfoliation/ dispersion method on $1 \mathrm{D}$ carbon, that is, on CNFs with a high edge-to-plane ratio. The HEM-assisted sonolysis of CNF-HEM provides, after $2 \mathrm{~h}$, a new carbon with a circular graphitic lattice covered with globin residues (Figures 5a,b and S8, Supporting Information). Locally, clearly exfoliated and separated graphitic segments can be observed as well. After the sedimentation step, the stable suspension contains almost $80 \%$ of pure product (Figure 5c). Its $\mathrm{CO}_{2}$ temperature-programmed desorption (TPD) analysis reveals that the type of oxygen groups is modified in the new carbon and the desorption process runs with a high content of lactone-type groups $\left(600-800{ }^{\circ} \mathrm{C}\right),{ }^{38}$ whereas the carboxylic group-related desorption areas (up to $450^{\circ}$ ) are quasi-absent compared to CNF before their exfoliation [Figure S9b; see also the Supporting Information for XPS, TGA, and Brunauer-Emmett-Teller (BET) analysis]. A significant content of highly electronegative oxygen-rich groups in CNFs is in accordance with a low combustion temperature, lower than that of CNF-HEM (Figure 5d). The difference in combustion temperature can be also related to the $\mathrm{Ni}$ residues which are absent in the case of CNF-HEM because they settled down during the sedimentation process.

The XPS results also reveal a decrease of the oxygen content after exfoliation (Figure $5 c$ and Table S1, Supporting Information). The high content of oxygen in the initial CNFs is indeed related to the acid/base treatments applied on the CNFs just after their synthesis to remove the remaining catalyst and support. This content decrease after the exfoliation/ 
dispersion process indicates some reduction. Because this phenomenon does not correlate with the data obtained for EG exfoliation, we suggest that this reduction can be related to the $\mathrm{Ni}$ catalyst traces, which are still present in CNFs as encapsulated species and difficult to remove by acid/base treatment. The exfoliation and cutting process in water allows the liberation of Ni traces acting next as a hydrogenation catalyst (trace amount of $\mathrm{Ni}$ does not disturb the activity of the exfoliated and cut CNFs in dehydrogenation reactions, as described below).

$\mathrm{CNF}-\mathrm{HEM}$ was tested as a metal-free catalyst for the selective dehydrogenation of ethylbenzene to styrene (ethylbenzene dehydrogenation, ED). The CNF subjected to sonolysis/mixing without the globin $\left(\mathrm{CNF}-\mathrm{H}_{2} \mathrm{O}\right.$, where only cutting and no "real" exfoliation occurs) and initial CNF (see Figure S8, Supporting Information, for TEM) were also tested. The results of the tests performed on time on stream reveal a higher conversion of ethylbenzene and a greater selectivity toward styrene on both "degraded" samples (32, 27, and 99\%, 99\% respectively) compared to initial CNFs (10 and 93\%, respectively) with the best conversion for exfoliated carbon (CNF-HEM) (Figure 5e). This confirms that the catalytic activity is not related to the potential traces of $\mathrm{N}$ and $\mathrm{Fe}$ in $\mathrm{CNF}-\mathrm{HEM}$, which would originate from HEM porphyrin $(\mathrm{Fe}$ and $\mathrm{N})$ and peptides $(\mathrm{N})$. None of these elements were detected by XPS, as expected, given the relative amount of these elements in the final sample. Fewpercent higher activity of CNF-HEM can be related to the higher exfoliation/dispersion degree of CNF-HEM compared to $\mathrm{CNF}-\mathrm{H}_{2} \mathrm{O}$ and the presence of globin as such. CNF-HEM is indeed a composite, where HEM protects the CNF from excessive agglomeration and stacking, thus increasing the accessibility of the active sites. The excellent catalytic performances of both catalysts are reflected in Figure $5 f$ through the benchmarking of their activities with those of Fe-based commercial catalysts and nanodiamonds. Excluding highly Ndoped carbons, ${ }^{39-41}$ CNF-HEM is one of the best metal-free unsupported catalysts known to date in the literature. ${ }^{22}$ In addition, taking into account the very harsh conditions for the synthesis of nanodiamonds (detonation, high pressure, and temperature) as well as their cost, CNF-HEM becomes an interesting alternative catalyst. Commonly, the activity of carbons in $\mathrm{ED}$ reaction is assigned to the quinone groups, ${ }^{42}$ and the presence of $\mathrm{C}=\mathrm{O}$-type groups in $\mathrm{CNF}-\mathrm{HEM}$ and $\mathrm{CNF}-\mathrm{H}_{2} \mathrm{O}$ is confirmed by XPS. However, more detailed studies on the structure/chemistry and catalytic activity of these systems will be provided in the future. To combine other potential structure-related advantages, CNF--HEM may be additionally doped with $\mathrm{N}$ and/or coupled/supported over other types of carbon.

To check the general relevance of the high HLB natural systems as exfoliation and/or dispersion agents coupled with ultrasonication/mixing forces, other different materials such as not only typical layered boron nitride (h-BN) and carbon nitride $\left(\mathrm{C}_{3} \mathrm{~N}_{4}\right)$ but also $\beta$-silicon carbide $(\mathrm{SiC})$ and $\mathrm{OD}$ nanodiamonds have been subjected to the BSA-assisted exfoliation/dispersion process. Their well-dispersed and stable colloids with a concentration of $8 \mathrm{~g} / \mathrm{L}$ have been successfully obtained (Supporting Information, Figures S9 and S10). Yet, detailed studies of their exfoliation and/or dispersion degrees are required.

\section{CONCLUSIONS}

Herein, we show that the use of natural systems of high HLB such as HEM, BSA, agar-agar, or maltodextrin and turbulence (mixing)/ultrasonication process in the exfoliation/dispersion of layered materials provides aqua colloids with great concentration. Two important items are revealed: large active natural systems possess high HLB and related o/w emulsification ability and the addition of mixing to the ultrasonication allows the unification the sound-reactant contact within the whole volume. Apart from the typical layered materials such as graphite, boron nitride, or carbon nitride, $\mathrm{SiC}$ and nanodiamonds have been tested. A concentration up to $47 \mathrm{~g} / \mathrm{L}$ is reached in the case of micrometer-sized well conductive FLG sheets derived from EG. Such obtained FLGs have low oxygen/defect content and high aspect ratio flakes, the latter being extremely important for the future composite or coating layer applications. The conductivity of FLG-HLB (FLG-BSA) composite reaches $10^{2} \mathrm{~S} / \mathrm{m}$ and can be increased to $10^{4}$ and $10^{5} \mathrm{~S} / \mathrm{m}$ by the annealing or removal of HLB system, respectively. If suitable, lower size and well-oxygen-decorated FLG flakes can be prepared by the strengthening of the exfoliation/dispersion conditions.

The process applied on CNFs results in exfoliation into a metal-free and $\mathrm{N}$-undoped catalyst, showing high performance in selective dehydrogenation of ethylbenzene to styrene.

Other successfully tested HLB systems include baobab karaya gum and gombo extracts. Many other "gastronomy, cosmetology, or drug" o/w emulsifiers including natural hydrocolloids (proteins, carbohydrates, and polysaccharides) or their mixtures, also extracted directly from the organic matter, are still to be explored, especially for high-concentration colloids. The rheological studies would help the establishment of optimal systems and ultrasonication/turbulence conditions (Reynolds number), ${ }^{43}$ which changes with the viscosity of colloids.

\section{EXPERIMENTAL SECTION}

Material Preparations. HEM from bovine blood, BSA, and myoglobin were purchased from Sigma-Aldrich. Maltodextrin was purchased from Myprotein. EG and graphite pellets were purchased from Carbon Lorraine and Timcal, respectively. Boron nitride was provided by Johnson Matthey Co. Nanodiamonds and silicon carbide were purchased from Carbodeon Co. Ltd. and SiCat SARL, respectively.

FLG-HLB, FLG-BSA, FLG-HEM, FLG-Myoglobin, FLGMaltodextrin, and FLG-Agar-Agar. (a) EG (12.8 g) and $1 \mathrm{~g}$ of HLB agent were added to $320 \mathrm{~mL}$ of distilled water. The ultrasonication was performed with an ultrasonic probe, Branson Digital Sonifier 450 , of $\sim 50 / 60 \mathrm{~Hz}$ frequency with an output intensity of $10 \%$ of $400 \mathrm{~W}$ and under continuous stirring for $2 \mathrm{~h}$. EG (4.5 g) and $0.45 \mathrm{~g}$ of BSA were next added, and everything was sonicated again for $1 \mathrm{~h}$. The formed suspension was left for sedimentation for $24 \mathrm{~h}$. The separated stable colloid has a concentration of around $47 \mathrm{~g} / \mathrm{L}$.

Other tested HLB were karaya gum and gombo extract. Karaya gum was obtained as dried powder from baobab tree. The HLB extract from gombo was obtained by cooking the vegetable in water at $80^{\circ} \mathrm{C}$ for $1 \mathrm{~h}$.

(b) Graphite pellets $(7.5 \mathrm{~g}$ ) and $0.75 \mathrm{~g}$ of BSA were added to $250 \mathrm{~mL}$ of distilled water. The ultrasonication was performed with an ultrasonic probe, Branson Digital Sonifier 450, of 50/ $60 \mathrm{~Hz}$ frequency with an output intensity of $10 \%$ of $400 \mathrm{~W}$ and under continuous stirring for $3 \mathrm{~h}$. The formed suspension was left for sedimentation for $24 \mathrm{~h}$. The separated stable colloid had a concentration of $7.7 \mathrm{~g} / \mathrm{L}$.

FLG-acid. The FLG-acid sample was prepared by the aqua regia treatment of FLG-BSA under reflux condition for $15 \mathrm{~h}$ in a 
round-bottom flask and subsequent purification with distillated water to neutral $\mathrm{pH}$, finalized by a drying step.

The CNFs were synthesized by a chemical vapor deposition method using a mixture of $\mathrm{C}_{2} \mathrm{H}_{6}$ and $\mathrm{H}_{2}$ and $\mathrm{Ni} / \mathrm{Al}_{2} \mathrm{O}_{3}$ as a growth catalyst.

The as-synthesized CNF was further purified of the nickel growth catalyst and the residual of the support by an acid and base treatment. The as-treated composite was further washed several times with deionized water until neutral $\mathrm{pH}$.

CNF-HEM. As a general procedure, $x \mathrm{mg}$ of CNF and $y \mathrm{mg}$ of protein were added to $z \mathrm{~mL}$ of distilled water with an $x / y / z$ ratio of 10:1:10. The ultrasonication was performed with an ultrasonic probe, Branson Digital Sonifier 450, of $\sim 50 / 60 \mathrm{~Hz}$ frequency with an output intensity of $10-20 \%$ of $400 \mathrm{~W}$ and under continuous stirring for $2 \mathrm{~h}$. The formed suspensions were left for sedimentation for $24 \mathrm{~h}$. The supernatants were next separated from the precipitates and eventually additionally submitted to the centrifugation process for $15 \mathrm{~min}$ at 2500 or $5000 \mathrm{rpm}$ (Thermo Scientific, Sorvall ST16 centrifuge).

$\mathrm{CNF}-\mathrm{H}_{2} \mathrm{O}$. $\mathrm{CNF}-\mathrm{H}_{2} \mathrm{O}$ was prepared following the same procedure but in the absence of the globin.

Catalytic Tests. The conditions of the catalytic tests, product analysis and conversion, and selectivity calculations are the same as ones previously reported. ${ }^{44}$ In brief, a steam-free dehydrogenation of ethylbenzene to styrene was carried out with $300 \mathrm{mg}$ of catalyst ( $\mathrm{CNF}-\mathrm{HEM}, \mathrm{CNF}-\mathrm{H}_{2} \mathrm{O}$, and $\left.\mathrm{CNF}\right)$, benchmarking catalysts and ethylbenzene $(2.8 \%$ in $\mathrm{He}$ ) flow of $30 \mathrm{~mL} / \mathrm{min}$ at $550{ }^{\circ} \mathrm{C}$ under ambient pressure. The reagents and products were analyzed online by gas chromatography (Perichrom, PR 2100) through flame ionization detection.

Characterization Tools. SEM was performed on a JEOL $2600 \mathrm{~F}$ instrument operated at an acceleration voltage of $15 \mathrm{kV}$ and an emission current of $10 \mathrm{~mA}$.

TEM was carried out on a JEOL $2100 \mathrm{~F}$ worked at a $200 \mathrm{kV}$ accelerated voltage, equipped with a probe corrector for spherical aberrations, and a point-to-point resolution of $0.2 \mathrm{~nm}$. Prior to the analysis, drops of the aqueous suspensions were deposited on holey carbon grids.

The XPS measurements were carried out in a UHV setup (base pressure $1 \times 10^{-9}$ mbar) equipped with a VSW class WA hemispherical electron analyzer (150 $\mathrm{mm}$ radius) with a multichannel electron detector. A monochromatic X-ray source (an $\mathrm{Al} \mathrm{K \alpha}$ anode operated at $240 \mathrm{~W}$ ) was used as the incident radiation. The XPS spectra were recorded in the fixed transmission mode using pass energies of $90 \mathrm{eV}$ for survey and $44 \mathrm{eV}$ for narrow scans. The Shirley method was employed for background subtraction, prior to the fitting procedure.

Raman spectra were recorded using a LabRAM ARAMIS Horiba Raman spectrometer equipment over the range of 500$4000 \mathrm{~cm}^{-1}$ at a laser excitation wavelength of $532 \mathrm{~nm}$. Prior to the measurements, the samples were deposited on a $\mathrm{SiO}_{2} / \mathrm{Si}$ substrate by the drop-casting of their suspension and carefully dried.

UV-vis spectra of the suspensions were recorded using a spectrophotometer equipped with a Peltier PTP1 system (PerkinElmer LAMBDA 35) at room temperature.

FPP sheet resistance $\left(R_{\mathrm{s}}\right)$ measurements were performed on thin carbon papers by the FPP method by inducing different current $(I)$ from $1 \mu \mathrm{A}$ to $1 \mathrm{~mA}$ through two external probes and measuring the voltage difference $(V)$ between two internal probes, with a Keithley 220 programmable current source coupled with a Hewlett-Packard 34401A multimeter. In the calculation of $R_{\mathrm{s}}$ from Ohm's law, a geometrical factor of the samples was considered. ${ }^{45}$

TPD analysis was carried out on a Micromeritics ASAP-2100 under vacuum from room temperature to $1000^{\circ} \mathrm{C}$ at a heating rate of $5{ }^{\circ} \mathrm{C} / \mathrm{min}$.

TGA was performed on a Setaram apparatus at an air flow rate of $25 \mathrm{~mL} / \mathrm{min}$ and a heating rate of $10{ }^{\circ} \mathrm{C} / \mathrm{min}$ from room temperature to $1000{ }^{\circ} \mathrm{C}$.

The specific surface area of the different samples was determined by the BET method using a Micromeritics TriStar sorptometer. The samples were outgassed at $250{ }^{\circ} \mathrm{C}$ under vacuum for $5 \mathrm{~h}$. Physisorption measurements were carried out using $\mathrm{N}_{2}$ as an adsorbent.

\section{ASSOCIATED CONTENT}

S Supporting Information

The Supporting Information is available free of charge on the ACS Publications website at DOI: 10.1021/acsomega.7b01311.

Additional analysis results (TEM, SEM, TGA, TPD, and optical images) (PDF)

\section{AUTHOR INFORMATION}

\section{Corresponding Author}

*E-mail: janowskai@unistra.fr. Phone: 33 (0)368852633 (I.J.).

ORCID 우

Izabela Janowska: 0000-0003-1785-2798

\section{Author Contributions}

I.J. is the principal investigator and project coordinator, who wrote the manuscript and carried out the conductivity measurements; H.B. carried out most of the experiments related to exfoliation and catalytic tests; L.T.-P. carried out most of the HEM-assisted exfoliation of EG and related initial experiments; C.P.-H. provided catalytic test facilities and carried out the testing of the $\mathrm{CNF}-\mathrm{H}_{2} \mathrm{O}$ catalyst; W.L. carried out the XPS experiments and related result treatments; W.B. conducted TEM measurements; and T.R. conducted SEM measurements.

\section{Notes}

The authors declare no competing financial interest.

\section{ACKNOWLEDGMENTS}

We acknowledge Corinne Ulhaq-Bouillet for TEM of CNFHEM. I. J. acknowledges CNRS for the financial support from "Programme de prématuration".

\section{REFERENCES}

(1) Paton, K. R.; Varrla, E.; Backes, C.; Smith, R. J.; Khan, U.; O’Neill, A.; Boland, C.; Lotya, M.; Istrate, O. M.; King, P.; et al. Scalable production of large quantities of defect-free few-layer graphene by shear exfoliation in liquids. Nat. Mater. 2014, 13, 624-630.

(2) Hernandez, Y.; Nicolosi, V.; Lotya, M.; Blighe, F. M.; Sun, Z.; De, S.; McGovern, I. T.; Holland, B.; Byrne, M.; Gun'Ko, Y. K.; et al. Highyield production of graphene by liquid-phase exfoliation of graphite. Nat. Nanotechnol. 2008, 3, 563-568.

(3) Ciesielski, A.; Samorì, P. Graphene via sonication assisted liquidphase exfoliation. Chem. Soc. Rev. 2013, 43, 381-398.

(4) Dreyer, D. R.; Todd, A. D.; Bielawski, C. W. Harnessing the chemistry of graphene oxide. Chem. Soc. Rev. 2014, 43, 5288-5301.

(5) Rozada, R.; Paredes, J. I.; Villar-Rodil, S.; Martínez-Alonso, A.; Tascón, J. M. D. Towards full repair of defects in reduced graphene oxide films by two-step graphitization. Nano Res. 2013, 6, 216-233.

(6) Qu, K.; Wu, L.; Ren, J.; Qu, X. Enzyme-directed pH-responsive exfoliation and dispersion of graphene and its decoration by gold nanoparticles for use as a hybrid catalyst. Nano Res. 2013, 6, 693-702. 
(7) Sharifi, F.; Bauld, R.; Ahmed, M. S.; Fanchini, G. Transparent and conducting graphene-RNA-based nanocomposites. Small 2012, 8, 699706.

(8) Liu, F.; Choi, J. Y.; Seo, T. S. DNA mediated water-dispersible graphene fabrication and gold nanoparticle-graphene hybrid. Chem. Commun. 2010, 46, 2844-2846.

(9) Ayán-Varela, M.; Paredes, J. I.; Guardia, L.; Villar-Rodil, S.; Munuera, J. M.; Díaz-González, M.; Fernández-Sánchez, C.; MartínezAlonso, A.; Tascón, J. M. D. Achieving Extremely Concentrated Aqueous Dispersions of Graphene Flakes and Catalytically Efficient Graphene-Metal Nanoparticle Hybrids with Flavin Mononucleotide as a High-Performance Stabilizer. ACS Appl. Mater. Interfaces 2015, 7, 10293-10307.

(10) Laaksonen, P.; Kainlauri, M.; Laaksonen, T.; Shchepetov, A.; Jiang, H.; Ahopelto, J.; Linder, M. B. Interfacial Engineering by Proteins: Exfoliation and Functionalization of Graphene by Hydrophobins. Angew. Chem., Int. Ed. 2010, 49, 4946-4949.

(11) Guan, G.; Zhang, S.; Liu, S.; Cai, Y.; Low, M.; Teng, C. P.; Phang, I. Y.; Cheng, Y.; Duei, K. L.; Srinivasan, B. M.; et al. Protein Induces Layer-by-Layer Exfoliation of Transition Metal Dichalcogenides. J. Am. Chem. Soc. 2015, 137, 6152-6155.

(12) Pattammattel, A.; Kumar, C. V. Kitchen Chemistry 101: Multigram Production of High Quality Biographene in a Blender with Edible Proteins. Adv. Funct. Mater. 2015, 25, 7088-7098.

(13) Unalan, I. U.; Wan, C.; Trabattoni, S.; Piergiovanni, L.; Farris, S. Polysaccharide-assisted rapid exfoliation of graphite platelets into high quality water-dispersible graphene sheets. RSC Adv. 2015, 5, 2648226490.

(14) Chabot, V.; Kim, B.; Sloper, B.; Tzoganakis, C.; Yu, A. High yield production and purification of few layer graphene by Gum Arabic assisted physical sonication. Sci. Rep. 2013, 3, 1378.

(15) K., P. K. M.; Shanthini, S.; Srivastava, C. Electrochemical exfoliation of graphite for producing graphene using saccharin. RSC Adv. 2015, 5, 53865-53869.

(16) Yu, Z.; Shi, Z.; Xu, H.; Ma, X.; Tian, M.; Yin, J. Green chemistry: Co-assembly of tannin-assisted exfoliated low-defect graphene and epoxy natural rubber latex to form soft and elastic nacre-like film with good electrical conductivity. Carbon 2017, 114, 649-660.

(17) Paredes, J. I.; Villar-Rodil, S. Biomolecule-assisted exfoliation and dispersion of graphene and other two-dimensional materials: a review of recent progress and applications. Nanoscale 2016, 8, 15389-15413.

(18) Yi, M.; Shen, Z. Kitchen blender for producing high-quality fewlayer graphene. Carbon 2014, 78, 622-626.

(19) Shen, H.; Zhang, L.; Liu, M.; Zhang, Z. Biomedical Applications of Graphene. Theranostics 2012, 2, 283-294.

(20) Gruner, G. Carbon nanotube transistors for biosensing applications. Anal. Bioanal. Chem. 2006, 384, 322-335.

(21) Gong, K.; Du, F.; Xia, Z.; Durstock, M.; Dai, L. Nitrogen-Doped Carbon Nanotube Arrays with High Electrocatalytic Activity for Oxygen Reduction. Science 2009, 323, 760-764.

(22) Zhang, J.; Su, D.; Zhang, A.; Wang, D.; Schlögl, R.; Hébert, C. Nanocarbon as Robust Catalyst: Mechanistic Insight into CarbonMediated Catalysis. Angew. Chem., Int. Ed. 2007, 46, 7319-7323.

(23) Terrones, M.; Botello-Méndez, A. R.; Campos-Delgado, J.; López-Urías, F.; Vega-Cantú, Y. I.; Rodríguez-Macías, F. J.; Elías, A. L.; Muñoz-Sandoval, E.; Cano-Márquez, A. G.; Charlier, J.-C.; et al. Graphene and graphite nanoribbons: Morphology, properties, synthesis, defects and applications. Nano Today 2010, 5, 351-372.

(24) Yu, Z.; Shi, Z.; Xu, H.; Ma, X.; Tian, M.; Yin, J. Green chemistry: Co-assembly of tannin-assisted exfoliated low-defect graphene and epoxy natural rubber latex to form soft and elastic nacre-like film with good electrical conductivity. Carbon 2017, 114, 649-660.

(25) Griffin, W. C. Classification of surface-active agents by "HLB". J. Soc. Cosmet. Chem. 1949, 1, 311-326.

(26) Ferran, A.; Mc Gee, H. Modern Gastronomy: A to Z, a Scientific and Gastronomic Lexicon; CRC Press Taylor \& Francis Group, 2010.

(27) Janowska, I.; Truong-Phuoc, L.; Ba, H.; Pham-Huu, C. BNT220716FR00, 10/11/2016.
(28) Nascentes, C. C.; Korn, M.; Sousa, C. S.; Arruda, M. A. Z. Use of ultrasonic baths for analytical applications: a new approach for optimisation conditions. J. Braz. Chem. Soc. 2001, 12, 57-63.

(29) Chivate, M. M.; Pandit, A. B. Quantification of Cavitation Intensity in Fluid Bulk. Ultrason. Sonochem. 1995, 2, S19-S25.

(30) Karagiannidis, P. G.; Hodge, S. A.; Lombardi, L.; Tomarchio, F.; Decorde, N.; Milana, S.; Goykhman, I.; Su, Y.; Mesite, S. V.; Johnstone, D. N.; et al. Microfluidization of Graphite and Formulation of Graphene-Based Conductive Inks. ACS Nano 2017, 11, 2742-2755.

(31) Vallés, C.; Abdelkader, A. M.; Young, R. J.; Kinloch, I. A. The effect of flake diameter on the reinforcement of few-layer graphenePMMA composites. Compos. Sci. Technol. 2015, 111, 17-22.

(32) Zhou, T. N.; Qi, X. D.; Fu, Q. The preparation of the poly(vinyl alcohol)/graphene nanocomposites with low percolation threshold and high electrical conductivity by using the large-area reduced graphene oxide sheets. eXPRESS Polym. Lett. 2013, 7, 747-755.

(33) Suslick, K. S.; Grinstaff, M. W. Protein microencapsulation of nonaqueous liquids. J. Am. Chem. Soc. 1990, 112, 7807-7809.

(34) Wong, M.; Suslick, K. S. Sonochemically Produced Hemoglobin Microbubbles. Mater. Res. Soc. Symp. Proc. 1994, 372, 89.

(35) Suslick, K. S. Sonochemistry. Science 1990, 247, 1439-1445.

(36) Hargreaves, A. E. An Overview of Surfactant Based Chemical Preparations Used in Everyday Life. Chemical Formulation; RSC: UK, 2003; pp 48-88.

(37) Ferrari, A. C.; Meyer, J. C.; Scardaci, V.; Casiraghi, C.; Lazzeri, M.; Mauri, F.; Piscanec, S.; Jiang, D.; Novoselov, K. S.; Roth, S.; et al. Raman Spectrum of Graphene and Graphene Layers. Phys. Rev. Lett. 2006, 97, 187401.

(38) Figueiredo, J. L.; Pereira, M. F. R. Synthesis and functionalization of carbon xerogels to be used as supports for fuel cell catalysts. J. Energy Chem. 2013, 22, 195-201.

(39) Liu, Y.; Ba, H.; Luo, J.; Wu, K.-H.; Nhut, J.-M.; Su, D. S.; PhamHuu, C. Structure-performance relationship of nanodiamonds@ nitrogen-doped mesoporous carbon in the direct dehydrogenation of ethylbenzene. Catal. Today 2017, DOI: 10.1016/j.cattod.2017.05.010.

(40) Ba, H.; Liu, Y.; Truong-Phuoc, L.; Duong-Viet, C.; Nhut, J.-M.; Nguyen, D. L.; Ersen, O.; Tuci, G.; Giambastiani, G.; Pham-Huu, C. Ndoped Food-grade derived 3D Mesoporous Foams as Metal-Free Systems for Catalysis. ACS Catal. 2016, 6, 1408-1419.

(41) Zhao, Z.; Dai, Y.; Lin, J.; Wang, G. Highly-Ordered Mesoporous Carbon Nitride with Ultrahigh Surface Area and Pore Volume as a Superior Dehydrogenation Catalyst. Chem. Mater. 2014, 26, 31513161.

(42) Pereira, M. F. R.; Orfão, J. J. M.; Figueiredo, J. L. Oxidative dehydrogenation of ethylbenzene on activated carbon catalysts 2 . Kinetic modeling. Appl. Catal., A 2000, 196, 43-54.

(43) Bakker, A.; Gates, L. E. Viscous Mixing. Chem. Eng. Prog. 1995, 91, 25-34.

(44) Thanh, T. T.; Ba, H.; Truong-Phuoc, L.; Nhut, J.-M.; Ersen, O.; Begin, D.; Janowska, I.; Nguyen, D. L.; Granger, P.; Pham-Huu, C. A few-layer graphene-graphene oxide composite containing nanodiamonds as metal-free catalysts. J. Mater. Chem. A. 2014, 2, 1134911357.

(45) Smits, F. M. Measurement of Sheet Resistivities with the FourPoint Probe. Bell Syst. Tech. J. 1958, 37, 711-718. 